\title{
Neutrinoless double beta decay and lepton number violation at the LHC
}

\author{
J.C. Held* and S.G. Kovalenkd \\ Universidad Técnica Federico Santa María, \\ Centro-Científico-Tecnológico de Valparaíso, \\ Casilla 110-V, Valparaíso, Chile \\ M. Hirsch $\ddagger$ \\ AHEP Group, Instituto de Física Corpuscular - C.S.I.C./Universitat de València \\ Edificio de Institutos de Paterna, Apartado 22085, E-46071 València, Spain \\ H. Päş \\ Fakultät für Physik, Technische Universität Dortmund, D-44221, Dortmund, Germany
}

\begin{abstract}
We compare the discovery potential of the LHC for lepton number violating (LNV) signals with the sensitivity of current and future double beta decay experiments, assuming $0 \nu \beta \beta$ decay is dominated by heavy particle exchange. We consider charged scalar, leptoquark and diquark mechanisms of $0 \nu \beta \beta$ decay, covering the $0 \nu \beta \beta$ decay operators with both, the smallest and largest, possible rates. We demonstrate, if $0 \nu \beta \beta$ decay were found with a half-life below $10^{26}-10^{27}$ ys a positive signal should show up at the LHC, except for some particular cases of the leptoquark mechanism, and vice versa, if the LHC does not find any hints for LNV, a "short-range" explanation for a finite $0 \nu \beta \beta$ decay half-life will be ruled out in most cases. We argue, if a positive LNV signal were found at the LHC, it is possible to identify the dominant contribution to $0 \nu \beta \beta$. Two different kinds of observables which could provide such "model discriminating" power are discussed: Different invariant mass peaks and the charge asymmetry.
\end{abstract}

\section{INTRODUCTION}

Neutrinoless double beta $(0 \nu \beta \beta)$ decay can be induced by the exchange of light particles such as Majorana neutrinos (long-range mechanism) or by heavy particles (short-range mechanism). The classical example of the latter is right-handed $\mathrm{W}$ boson and heavy neutrino exchange, which occurs in left-right symmetric extensions of the standard model [1]. A number of other examples of such "short-range" contributions to $0 \nu \beta \beta$ decay have been discussed in the literature (for a review see [2]), but only very recently the general decomposition for the $0 \nu \beta \beta$ decay operator, $\mathcal{O}_{d=9}^{0 \nu \beta \beta}=\frac{1}{M^{5}} \bar{u} \bar{u} d d \bar{e} \bar{e}$ (where $M$ has dimension of mass) in terms of renormalizable vertices connected by heavy virtual particles, has been given in [3]. Interestingly, several new diagrams for $0 \nu \beta \beta$ decay were identified in this work, many of which require quite exotic particles such as diquarks or colored fermions with fractional charges. Common to all of the shortrange contributions is that masses of order $M \sim(1-5)$ $\mathrm{TeV}$ are needed for a $0 \nu \beta \beta$ decay half-life in the range of $T_{1 / 2}^{0 \nu \beta \beta} \sim\left(10^{25}-10^{27}\right)$ ys in either ${ }^{76} \mathrm{Ge}$ or ${ }^{136} \mathrm{Xe}$.

The very same diagrams which lead to $0 \nu \beta \beta$ decay can lead to LHC signals with like-sign dileptons plus (at least) two jets in the final state [4, [5]. The ATLAS [ and the CMS [7] collaborations have published searches for such events. (Disregarding flavor, we will refer to these events as "eejj-like".) ATLAS and CMS then interpret their null result in terms of the left-right symmetric model as lower limits on the mass of the right-handed gauge boson $m_{W_{R}} \gtrsim(2.5-2.9) \mathrm{TeV}$ [7], depending on the mass of the right-handed neutrino $m_{N}$ and assuming the coupling $g_{R}$ of $W_{R}$ to fermions is equal to the standard model coupling $g_{L}$. Note that [6] provides separate limits for opposite-sign (OS) and same-sign (SS) dileptons, while CMS provides only combined limits. While, of course, the search for OS and SS is experimentally very similar, obviously only SS-type events are a proof for the discovery of lepton number violation (LNV).

In this paper we discuss how searches for LNV signals at the LHC compare with the sensitivities of current and future $0 \nu \beta \beta$ decay experiments. We do not limit ourselves to a particular model of LNV, but use a general decomposition of $\mathcal{O}_{d=9}^{0 \nu \beta \beta}$. We show that a nonobservation of eejj-like events in the future LHC run at $\sqrt{s}=14$ $\mathrm{TeV}$ would rule out all but the pure leptoquark decompositions as the dominant contribution to $\mathcal{O}_{d=9}^{0 \nu \beta \beta}$. Perhaps even more interesting is that, if a positive LNV signal were found at the LHC, it is possible to identify the dominant contribution to $0 \nu \beta \beta$. We discuss two types of observables which could provide such "model discriminating" power: The construction of different invariant mass combinations and the ratio of positronlike to electronlike events. We will call the latter the "charge asymmetry".

\section{SHORT-RANGE DOUBLE BETA DECAY}

Double beta decay can be induced by exchange of scalars or vectors with or without an accompanying virtual fermion. Since the results for scalars and vectors 
are qualitatively very similar, we will concentrate on the scalar case only. Also, there are two basic topologies of the diagrams contributing to $0 \nu \beta \beta$ decay. Here, we limit ourselves to the discussion of Topology I (scalar-fermionscalar exchange) shown in fig. (11). The results for Topology II (triple-scalar exchange coupled to the trilinear vertex) will be presented elsewhere [8]. There are in total 18 different possible decompositions ("diagrams") in Topology I that can contribute to the $0 \nu \beta \beta$ decay operator, $\mathcal{O}_{d=9}^{0 \nu \beta \beta}$. Depending on the color and $S U(2)_{L}$ transformation properties of the internal fermion and bosons, each decomposition can be realized in several ways, see below. Twelve decompositions involve fractionally charged (and colored) fermions, and were discussed for the first time in [3]. The remaining six possibilities have all been discussed before in the literature in the context of concrete models, most notably the mass mechanism or R-parity violating supersymmetry [9, 10].

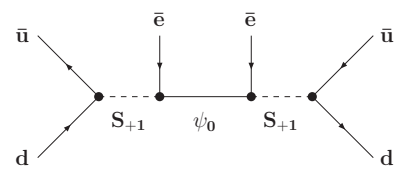

(a)

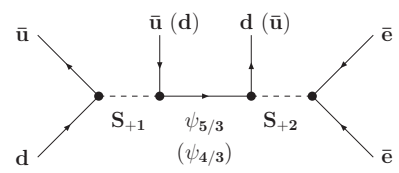

(c)

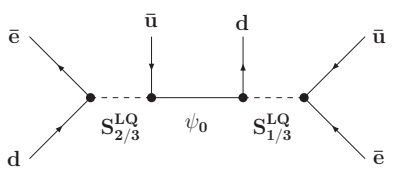

(b)

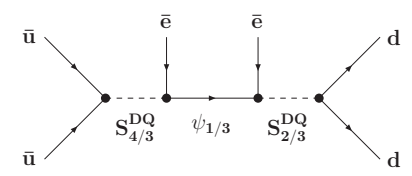

(d)
FIG. 1: Example diagrams of Topology I for short-range double beta decay, see text: (a) charged scalar $S_{+1}$ - neutral fermion $\psi_{0}$ - charged scalar $S_{+1}$; (b) leptoquark $S_{2 / 3}^{L Q}$ - neutral fermion $\psi_{0}$ - leptoquark $S_{1 / 3}^{L Q}$; (c) charged scalar $S_{1}$ - charged colorful fermion $\psi_{q}$ - charged scalar $S_{+2} ;$ (d) diquark $S_{4 / 3}^{D Q}-$ charged colorful fermion $\psi_{1 / 3}-\operatorname{diquark} S_{2 / 3}^{D Q}$

In Fig. 1 we show some example diagrams for $0 \nu \beta \beta$ decay. These examples were chosen such that all six different bosons $\left(S_{+1}, S_{+2}, S_{2 / 3}^{L Q}, S_{1 / 3}^{L Q}, S_{4 / 3}^{D Q}\right.$ and $\left.S_{2 / 3}^{D Q}\right)$ and all four types of fermions $\left(\psi_{0}, \psi_{1 / 3}, \psi_{4 / 3}\right.$ and $\left.\psi_{5 / 3}\right)$, which can contribute to $0 \nu \beta \beta$ decay, appear at least once each in the figure. We use the notation $S_{q}^{x}$ (and $\psi_{q}$ ) to denote a scalar (fermion) with electric charge $q$ and the superscript $x=D Q$ or $x=L Q$ to differentiate between diquarks and leptoquarks. Note that replacing $S_{+1}$ in Fig.1(a) on both sides by a standard model $\mathrm{W}$ boson and interpreting $\psi_{0}$ as a neutrino would lead to the classical mass mechanism, and diagrams of the type shown in Fig.1(b) appear, for example, in R-parity violating supersymmetry, while Figs.1(c) and 1(d) are examples of the new type of diagrams.

Integrating out the heavy particles in all cases leads to the $\mathcal{O}_{d=9}^{0 \nu \beta \beta}$ operator and to an amplitude of $0 \nu \beta \beta$ decay

$$
\mathcal{A}^{0 \nu \beta \beta} \propto \frac{g_{1} g_{2} g_{3} g_{4}}{m_{S_{i}}^{2} m_{\psi_{q}} m_{S_{j}}^{2}} \equiv \frac{g_{\text {eff }}^{4}}{M_{\text {eff }}^{5}} .
$$

We denote the couplings as $g_{1}-g_{4}$, arbitrarily read from left to right in the diagrams. All of these are in principle different free parameters in general. $m_{S_{i}}, m_{S_{j}}$ and $m_{\psi_{q}}$ are the masses of the bosons and the fermion, respectively, not necessarily $m_{S_{i}}=m_{S_{j}}$.

While $\mathcal{A}^{0 \nu \beta \beta}$ depends on the particle physics parameters always in the form of Eq.(1), the nuclear matrix elements (and thus the half-life) depend on the chirality of the "outer" fermions as well as the color representation of the "inner" particles in the diagram. The recent paper [3] provides a complete list of operators (for short-range $0 \nu \beta \beta$ decay with scalars), which, once numerical values for the nuclear matrix elements are taken from, for example [2], can be directly converted into calculated halflives, using $T_{1 / 2}^{0 \nu \beta \beta}=\mathcal{C}_{i}^{2}\left(\mathcal{M}_{i}^{N u c l}\right)^{2} G$, where $G$ stands for a phase space integral, $\mathcal{M}_{i}^{N u c l}$ for the nuclear matrix element and $\mathcal{C}_{i}$ is the coefficient of the dimension-9 operator made dimensionless by a numerical factor of $2 m_{P} / G_{F}^{2}$. Current limits from ${ }^{76} \mathrm{Ge} \mathrm{[11]} \mathrm{and}{ }^{136} \mathrm{Xe}$ [12, 13], both of the order of $T_{1 / 2}^{0 \nu \beta \beta} \gtrsim 10^{25} \mathrm{ys}$, correspond to roughly $M_{\text {eff }} \gtrsim(1.2-3.2) g_{\text {eff }}^{4 / 5} \mathrm{TeV}$, depending on decomposition. These limits depend on nuclear matrix elements, for which a typical uncertainty of a factor of $\sim 2$ is usually quoted in the literature.

\section{SENSITIVITY: LHC VERSUS $0 \nu \beta \beta$ DECAY}

The number of eejj-like events at the LHC in general depends on a different combination of couplings and masses than the $0 \nu \beta \beta$ decay amplitude. Here, for brevity, we will discuss only the "symmetric decompositions", see below. We stress, however, that we have checked that despite being a special case, the results presented below still cover both the most optimistic and the most pessimistic scenario for the LHC. A systematic case-by-case study covering all decompositions and cases in detail will be presented elsewhere [8]. In "symmetric" decompositions, such as Fig.1(a), couplings are pairwise equal, i.e. $g_{1}=g_{4}$ and $g_{2}=g_{3}$. Assuming that such an equality holds at least approximately, in the small width approximation we can write the number of events at the LHC as

$$
\begin{aligned}
\#(e e j j) / \mathcal{L} & =\sigma\left(p p \rightarrow S_{i}\right) \times \operatorname{Br}\left(S_{i} \rightarrow \text { eejj }\right) \\
= & F_{S_{i}}\left(\sqrt[4]{\frac{\left(M_{\left.\mathrm{eff}\left(S_{i}\right)\right)^{5}}\right.}{m_{\psi_{q}}}}\right) g_{\text {eff }}^{4} \operatorname{Br}^{e f f}\left(S_{i} \rightarrow e e j j\right)
\end{aligned}
$$

It is assumed here that $m_{S_{i}}>m_{\psi_{q}}$, such that both particles are on shell. Thus, our analysis is not valid for 
$m_{S_{i}}<m_{\psi_{q}}$. Also, we have rewritten $F_{S_{i}}\left(m_{S_{i}}\right)=\sigma(p p \rightarrow$ $\left.S_{i}\right) / g_{1}^{2}$ and $\operatorname{Br}^{e f f}\left(S_{i} \rightarrow e e j j\right)=\operatorname{Br}\left(S_{i} \rightarrow e e j j\right) / g_{2}^{2}$. While $\operatorname{Br}\left(S_{i} \rightarrow e e j j\right)$ is fixed for a given decomposition, once all couplings and masses are fixed, here we simply treat $\operatorname{Br}^{e f f}\left(S_{i} \rightarrow e e j j\right)$ as a free number smaller than one. Note, however, that in the limit where all couplings are equal (and $\left.m_{\psi_{q}}=0\right) \operatorname{Br}\left(S_{+1} \rightarrow e^{+} e^{+} j j\right)=$ $\operatorname{Br}\left(S_{+1} \rightarrow e^{+} e^{-} j j\right) \simeq 1 / 8$ for $S_{+1}$. In the LQ case, the LQ is produced in association with a lepton, i.e. we calculate $\sigma\left(p p \rightarrow S_{i}+e\right) \times \operatorname{Br}\left(S_{i} \rightarrow e j j j\right)$, see next section. Single LQ production at the LHC is mainly through $G+q \rightarrow S_{q}^{L Q}+e$, i.e. to the corresponding diagrams in Fig. (11) one has to attach a gluon to the initial quark. We have used CalcHEP [14] and MadGraph 5 [15] to calculate the production cross sections for $S_{+1}, S_{q}^{L Q}$, and $S_{q}^{D Q}$ at the LHC. We have compared our results with the literature [16-18] and found quite good agreement in all cases.
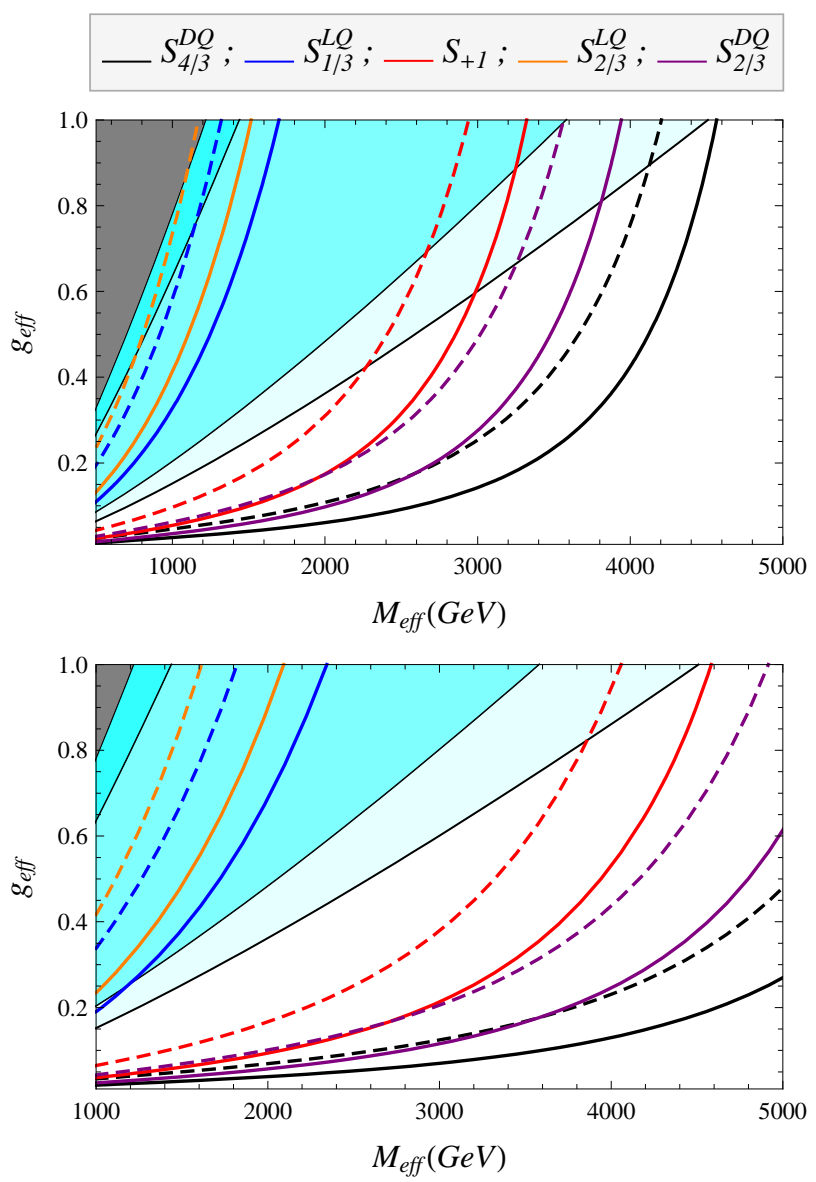

FIG. 2: Sensitivity limits for the LHC for production of five different scalar bosons compared to current and future double beta decay experiments. (a) Top: $m_{\psi_{q}}=200 \mathrm{GeV}$; (b) bottom: : $m_{\psi_{q}}=1 \mathrm{TeV}$, see text.

In Fig. (22) we then plot the compared sensitivities of $0 \nu \beta \beta$ decay and the LHC for five different cases, using Eq. (2). The more complicated "asymmetric" case, with more than one scalar mass and all couplings differ- ent, will be presented elsewhere [8] but leads to the same conclusions. For the LHC we show the expected sensitivity, assuming less then three signal events in $300 \mathrm{fb}^{-1}$ of statistics. Note, however, that our conclusions are not affected even if this assumed upper limit is as large as ten signal events. We plot for two values of $\operatorname{Br}^{e f f}(S \rightarrow e e j j)$, i.e $10^{-2}$ (dashed lines) and $10^{-1}$ (solid lines) in the plane $g_{e f f}$ versus $M_{e f f}$, for two different values of $m_{\psi}=200$ $\mathrm{GeV}$ (top) and $m_{\psi}=1 \mathrm{TeV}$ (bottom). The different color codes of the lines correspond to the five different scalar bosons, that can be singly produced at the LHC, namely $S_{+1}, S_{2 / 3}^{L Q}, S_{1 / 3}^{L Q}, S_{4 / 3}^{D Q}$ and $S_{2 / 3}^{D Q}$. Note that $S_{+1}$ is very similar to the well-known case of $W_{R}$ production in LR symmetry and $S_{2 / 3}^{L Q}$ and $S_{4 / 3}^{D Q}$ correspond to the most pessimistic and most optimistic scenario for the LHC.

In addition, Fig. (2) shows four different cases for current and future limits from $0 \nu \beta \beta$ decay. The dark gray area is the currently excluded part of parameter space from nonobservation of ${ }^{136} \mathrm{Xe}$ decay with $T_{1 / 2}^{0 \nu \beta \beta} \geq$ $1.6 \times 10^{25}$ ys [12] assuming $0 \nu \beta \beta$ decay is caused by the operator with the smallest rate and thus corresponds to the most pessimistic case for the sensitivity of $0 \nu \beta \beta$ decay. The three blue areas are (from left to right) the smallest rate, but for a limit of $T_{1 / 2}^{0 \nu \beta \beta} \geq 10^{26} \mathrm{ys}$, the largest rate with $T_{1 / 2}^{0 \nu \beta \beta} \geq 10^{26}$ ys, and the largest rate with $T_{1 / 2}^{0 \nu \beta \beta} \geq 10^{27}$ ys. The lightest area to the right therefore corresponds to the most optimistic reach for $0 \nu \beta \beta$ decay in the foreseeable future.

As can be seen, with the exception of the LQ cases, the LHC at $\sqrt{s}=14 \mathrm{TeV}$ will be more sensitive than $0 \nu \beta \beta$ decay experiments as probe for LNV. For the LQ case, the LHC covers the region explored by future $0 \nu \beta \beta$ decay experiments only partially. Note that decompositions with one LQ and one $S_{+1}$ or one $S_{q}^{D Q}$ will be similarly constrained by LHC searches as the "symmetric" $S_{+1}$ or $S_{q}^{D Q}$ case.

\section{DISTINGUISHING LNV MODELS}

As long as LHC experiments provide only upper limits on LNV signals, conversion to model-dependent exclusion plots is, apart from different numerical factors, quite similar in all cases. However, if a positive signal is observed in the future, the LHC has the potential to differentiate between different LNV models. Probably the two most promising observables to do so are the different invariant mass peaks and the charge asymmetry.

\section{A. Invariant mass peaks}

One can divide the discussion into (i) s-channel production of either $S_{+1}$ or $S_{q}^{D Q}$, (ii) associated $S_{q}^{L Q}+e$ 
production and (iii) pair production of a colored fermion $\psi_{q}$.

(i) All decompositions with s-channel production of $S_{+1}$ or $S_{q}^{D Q}$ lead to the final state eejj with a mass peak at $m_{e e j j}^{2}=m_{S}^{2}$. Consider first the simplest case, Fig.1(a). This case is equal to the well-known LR-symmetric case: On-shell production of $S_{+1}$ decaying to $\psi_{0}+e$, with $\psi_{0} \rightarrow e j j$ via off-shell $\left(S_{+1}\right)^{*}$ leads to two invariant mass peaks $m_{e e j j}^{2}=m_{S_{+1}}^{2}$ and $m_{e_{2} j j}^{2}=m_{\psi_{0}}^{2}$. On the other hand, $m_{e e}^{2}$ has a broad distribution. Compare this to, for example, Fig.1(c). Here, still $m_{e e j j}^{2}=m_{S_{+1}}^{2}$, but $m_{e e j_{2}}^{2}=m_{\psi_{q}}^{2}$ and since $m_{S_{+2}}<m_{\psi_{q}}$ is possible, a third peak could show up at $m_{e e}^{2}=m_{S_{+2}}^{2}$.

(ii) $S_{q}^{L Q}$ production is different, since it is produced in association with a lepton, i.e. there are (at least) three jets in the final state and $m_{e e j j j}^{2}$ does not show a peak, while $m_{e_{2} j j j}^{2}=m_{S_{i}^{L Q}}^{2}$. Again, different subsystems of $e_{2} j j j$ have peaks, depending on mass ordering and exact decomposition, but in the $S_{q}^{L Q}$ case there never is a peak in the $m_{e e}^{2}$ distribution.

(iii) As mentioned above, 12 decompositions have fractionally charged fermions, all of which have to be colored. These fermions can be pair produced through gluon-gluon fusion and the final state of interest to us is $e e+4 j$. The events of this type will have a threshold of $m_{e e j j j j}^{2}=4 m_{\psi_{q}}^{2}$ and their subsystems either eej and $j j j$ or $e_{1} j_{1} j_{2}$ and $e_{2} j_{3} j_{4}$ will show mass peaks at $m_{\psi_{q}}^{2}$.

Finally, there are decompositions with both diquarks and leptoquarks with in general different masses, which could lead to events with $2 j, 3 j$ and $4 j$ having mass peaks in different subsystems and at different values.

The following comments might be in order. First, charged scalar and both diquarks leave the "classical" mass peak in eejj. The experimental sensitivity to this mass peak and the experimental resolution for it is known, and has been simulated by both ATLAS [6] and CMS [7]. The different subsystems, that we discussed as "model" discriminators are then found within the set of those events. Thus, their energy spread is determined by and smaller than the one of the full system eejj. The only mass peaks, which are indeed different, are the ones produced by leptoquarks, where the events eejjj are more spread out in energy. For obtaining the exact number of events, which are needed for discovery, a MonteCarlo simulation will be helpful and will be carried out elsewhere.

\section{B. Charge asymmetry}

Define the charge asymmetry as the ratio of the number of events

$$
x_{C A}=\#\left(e^{+} e^{+}\right) / \#\left(e^{-} e^{-}\right) .
$$

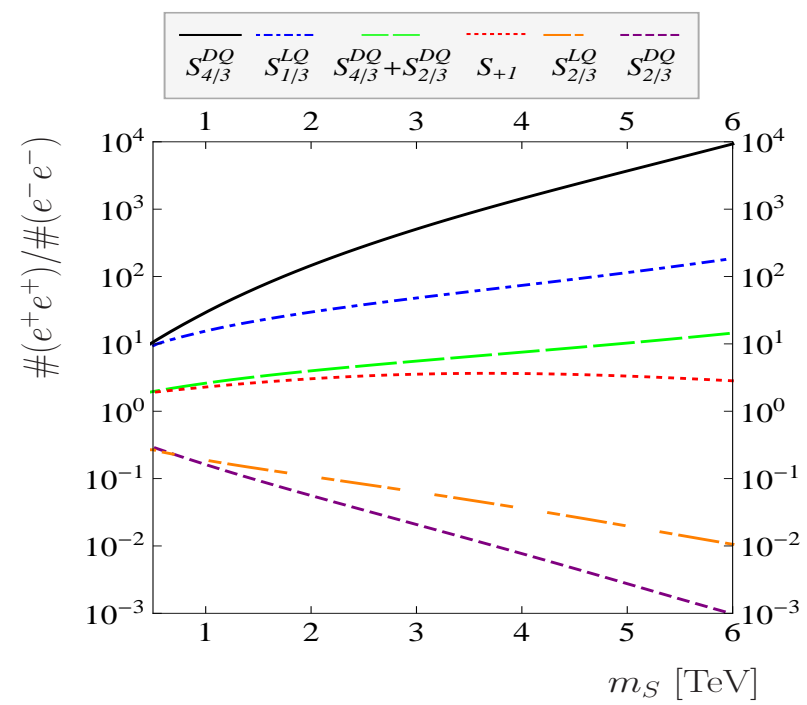

FIG. 3: Charge asymmetry, i.e. ratio of positronlike to electronlike events as a function of the boson mass for different kinds of scalars.

Consider first the simplest case of Fig.1(a). With only one boson and two pairwise equal couplings $\left(g_{1}=g_{4}\right.$ and $\left.g_{2}=g_{3}\right) x_{C A}$ is simply determined by the ratio of production cross section for $\sigma\left(p p \rightarrow S_{+1}\right)$ divided by $\sigma\left(p p \rightarrow S_{-1}\right)$. Since the number of $u$ quarks is different from the number of $d$ quarks in the proton, the two cross sections are not the same and one expects to see more events with $e^{+} e^{+}$than $e^{-} e^{-}$. More generally, $x_{C A}$ is a function of all masses and couplings, but even in the limit where couplings are equal, different $0 \nu \beta \beta$ decay decompositions have different values for $x_{C A}$.

Figure (3) shows the theoretically expected value of $x_{C A}$ for six different cases in the equal coupling limit. These cases shown do not (always) directly correspond to a particular decomposition, but represent extreme limits, where the scalar identified by the color code gives the dominant contribution to $x_{C A}$. The case $S_{2 / 3}^{D Q}+S_{4 / 3}^{D Q}$ assumes that both diquarks have the same mass and couplings and thus is just the average of the two individual cases. If both $S_{1 / 3}^{L Q}$ and $S_{2 / 3}^{L Q}$ contribute equally to the $0 \nu \beta \beta$ decay rate (case not shown), the two asymmetries average to a number close to, but not equal to the case of $S_{+1}$. Note that the case for $S_{+1}$ is equal to the expectations in the left-right symmetric model. In this cases, the charge asymmetry varies only weakly from $x_{C A} \simeq(2-4)$ in the mass range shown. However, as the figure demonstrates, in other cases much larger (and much smaller) $x_{C A}$ are possible. Experimentalists should therefore provide data sets separately for positively and negatively charged leptons, in order to cover all LNV models. Finally, note that for the LQ case we show curves up to $6 \mathrm{TeV}$, but realistically (compare Fig. (2)) for LQs the LHC will not have sufficient sensitivity to find any events 
for LQ masses larger than roughly $2 \mathrm{TeV}$.

\section{CONCLUSIONS}

We have compared the sensitivity of the LHC experiments for LNV signals with current and future sensitivities of $0 \nu \beta \beta$ decay experiments. The LHC has large discovery potential for general LNV violating models. In case a positive signal is found in the future at the LHC, we have proposed two types of observables, which allow distinguishing different LNV models: (i) particular sets of invariant mass peaks and (ii) the ratio of positron to electron same-sign dilepton events.

\section{Acknowledgements}

This work was supported by UNILHC PITN-GA2009-237920 and by the Spanish MICINN Grants No, FPA2011-22975 and No. MULTIDARK CSD2009-00064, by the Generalitat Valenciana (Prometeo/2009/091), by Fondecyt Grants No. 11121557 and No. 1100582, and CONICYT Projects No. 791100017 and No. CONICYT/DFG-648. H.P. was supported by DGF Grant No. PA 803/6-1.

* Electronic address: juan.heloherrera@gmail.com

† Electronic address: sergey.kovalenko@usm.cl

¥ Electronic address: mahirsch@ific.uv.es

$\S$ Electronic address: heinrich.paes@uni-dortmund.de

[1] R. N. Mohapatra and G. Senjanovic, Phys. Rev. D 23, 165 (1981); Riazuddin, R. E. Marshak, and R. N. Mohapatra, Phys.Rev. D 24, 1310 (1981).
[2] F. F. Deppisch, M. Hirsch and H. Päs, J. Phys. G 39, 124007 (2012).

[3] F. Bonnet, M. Hirsch, T. Ota, and W. Winter, JHEP 1303, 055 (2013).

[4] W.-Y. Keung and G. Senjanovic, Phys.Rev.Lett. 50, 1427 (1983).

[5] B. C. Allanach, C. H. Kom, and H. Päs, Phys.Rev.Lett. 103, 091801 (2009).

[6] G. Aad et al. (ATLAS Collaboration), Eur.Phys.J. C 72, 2056 (2012).

[7] CMS Collaboration, (2012), PAS EXO-12-017.

[8] J. C. Helo, M. Hirsch, H. Päs and S. G. Kovalenko, arXiv:1307.4849 [hep-ph].

[9] R. N. Mohapatra, Phys. Rev. D 34, 3457 (1986).

[10] M. Hirsch, H. V. Klapdor-Kleingrothaus and S. G. Kovalenko, Phys. Rev. Lett. 75, 17 (1995).

[11] H. Klapdor-Kleingrothaus et al., Eur.Phys.J. A 12, 147 (2001).

[12] M. Auger et al. (EXO Collaboration), Phys.Rev.Lett. 109, $03250 \overline{5(2012) \text {. }}$

[13] A. Gando et al. (KamLAND-Zen Collaboration), Phys. Rev. Lett. 110, 062502 (2013).

[14] A. Pukhov, arXiv:hep-ph/0412191 A. Belyaev, N.D. Christensen and A. Pukhov, Comput. Phys. Commun. 184, 1729 (2013).

[15] J. Alwall, M. Herquet, F. Maltoni O. Mattelaer, and T. Stelzer, JHEP 1106, 128 (2011).

[16] A. Ferrari, et al., Phys. Rev. D 62, 013001 (2000).

[17] A. Belyaev, C. Leroy, R. Mehdiyev and A. Pukhov, JHEP 0509, 005 (2005).

[18] T. Han, I. Lewis and Z. Liu, JHEP 1012, 085 (2010). 